\title{
ETUDE CRITIQUE DE L'EXPLORATION HEMODYNAMIQUE PER-ANESTHESIQUE
}

\author{
P. Stieglitz et P. Girardet*
}

Chaque anesthésiste doit un jour planifier la surveillance hémodynamique de ses patients les plus vulnérables et choisir les substances les moins dépressives pour les rendre opérables.

Dans les manuels, tout parait bien défini, mais depuis quelques années, les avis évoluent et deviennent nuancés. Les articles récents, très documentés, de Patschke,, Merin, ${ }^{2}$ Dauchot, ${ }^{3}$ Byrick, ${ }^{4}$ Filner $^{5}$ ont des conclusions ambiguës et, par exemple, ne permettent pas de trancher sur la valeur des indices de temps systolique dans l'appréciation de la contractilité myocardique, ou sur le bien-fondé de l'administration d'enflurane, d'étomidate, d'alfatésine, de morphiniques chez les coronariens.

Les retombées pratiques de ces excellents travaux ne sont pas immédiates, tant il est vrai, comme l'affirme Sonnenblick ${ }^{6}$ que pour un clinicien "le cœur est bon ou mauvais" et pour un fondamentaliste, il est "l'objet de son observation attentive".

Toute la difficulté de l'évaluation hémodynamique vient: du complet auto-asservissement du système cardio-vasculaire; de l'interdépendance des variables et de l'aspécificité des indices de mesures obtenus.

I. LESYSTEME CIRCULATOIRE EST ASSERVI A LUI-MEME; TOUTE ACTION SUR L'UNE DES TROIS COMPOSANTES ALTERE L'UNE OU LES DEUX AUTRES

Sur le schéma $n^{\circ} 1$, représentant trois roues reliées entre elles par des courroies de transmission, une action sur la consommation tissulaire entraine une réaction dans la perfusion périphérique et une modification de la fonction cardiaque.

Un exemple simple en est donné par l'injection de nitro-prussiate de soude qui diminue les résistances périphériques, ne modifie pas la consommation d'oxygène mais décroit très notablement le travail systolique d'opérés (schéma $n^{\circ} 2$ ) de neurochirurgie.

* Département d'Anesthésie-Réanimation, Centre Hospitalier, Universitaire de Grenoble, 38700 La Tronche, France.

Canad. Anaesth. Soc. J., vol. 25, no. 3, May 1978
Le muscle cardiaque est lui-même un soussystème asservi. Une réduction du travail cardiaque diminue la $\mathrm{VO}_{2}$ et la perfusion coronaire et vice-versa (schéma $n^{0} 3$ ).

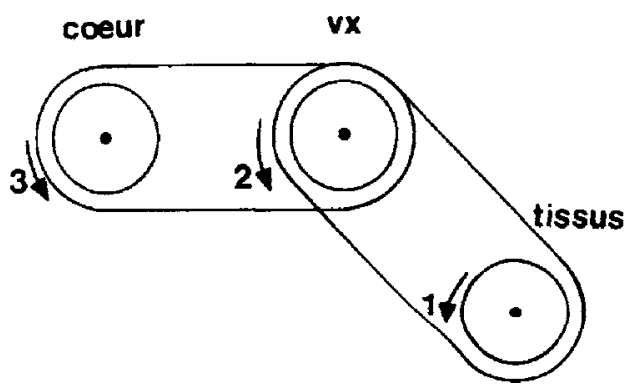

Schema N"1. Propulsion, distribution, consommation sont étroitement liées. Un tour de roule en I provoque des ajustements en 2 et en 3 . Il y a quelque chose de parfaitement mécanique dans ces enchainements.

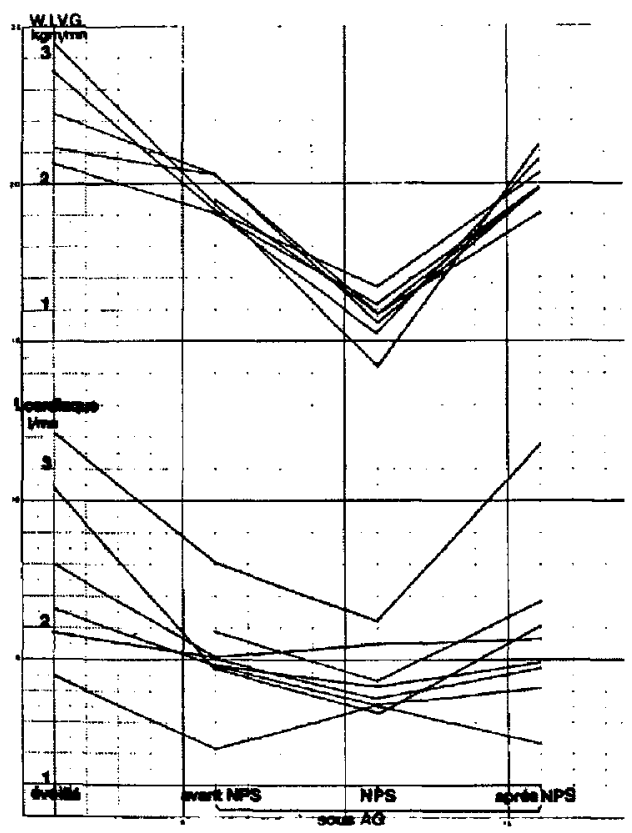

SCHEMA N*2. Action du nitroprussiate sur le débit cardiaque et le travail systolique. (in NANDAN - 31). 


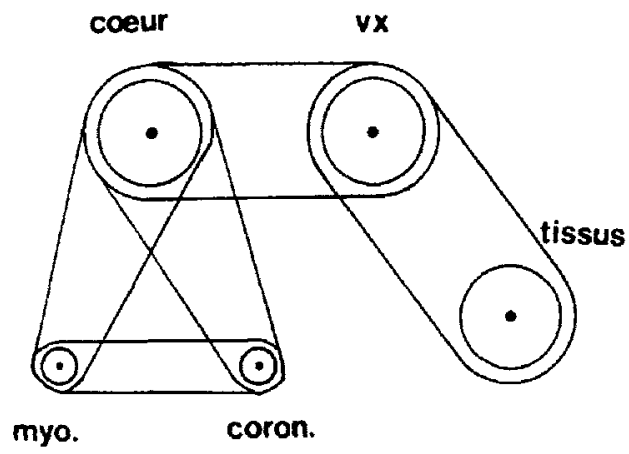

SChema No 3. Si le cour augmente son travail, la perfusion coronaire et la $\mathrm{VO}_{2}$ myocardique s'accroissent.

Le rôle du sympathique est de "mobiliser" le système circulatoire dans son ensemble et selon un certain mode, il n'a pas d'effet isolé sur la contractilité, le tonus vasomoteur ou la fréquence cardiaque.

Il résulte de ces dépendances réciproques qu'un agent pharmacodynamique semble agir à trois niveaux en même temps, sans que l'on puisse toujours déterminer le point d'impact initial. Par example, les halogénés diminuent les résistances systémiques, le travail cardiaque, la post-charge, la contractilité. Si l'on n'avait observé sur des préparations de muscle cardiaque ou par des artifices expérimentaux l'effet direct des halogénés sur le myocarde, on pourrait toujours douter de la spécificité de cette action (schéma n ${ }^{\circ} 4$ ).

Cette déduction théorique est confirmée par des enregistrements simultanés polygraphiques en cours de "crise" hémodynamique.

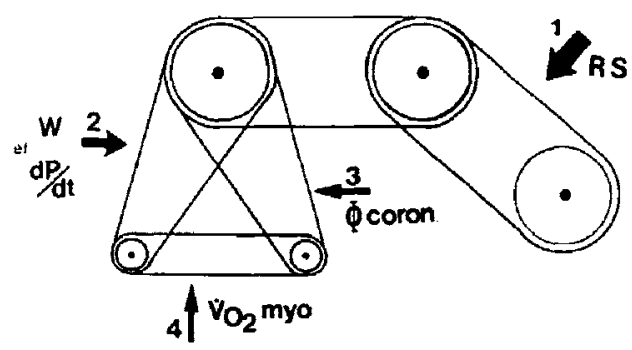

Schema No $N^{\circ}$. Les anesthésiques diminuent en général la consommation globale d'oxygène et les résistances périphériques. De ce fait, le travail cardiaque diminue puis la perfusion coronaire et la $\mathrm{Vo}_{2}$ myocardique. Si l'on n'avait montré in vitro que ces substances ont un effet direct sur le tissu cardiaque, il serait risqué de parler de "dépression myocardique" liée aux halogénés, par exemple.

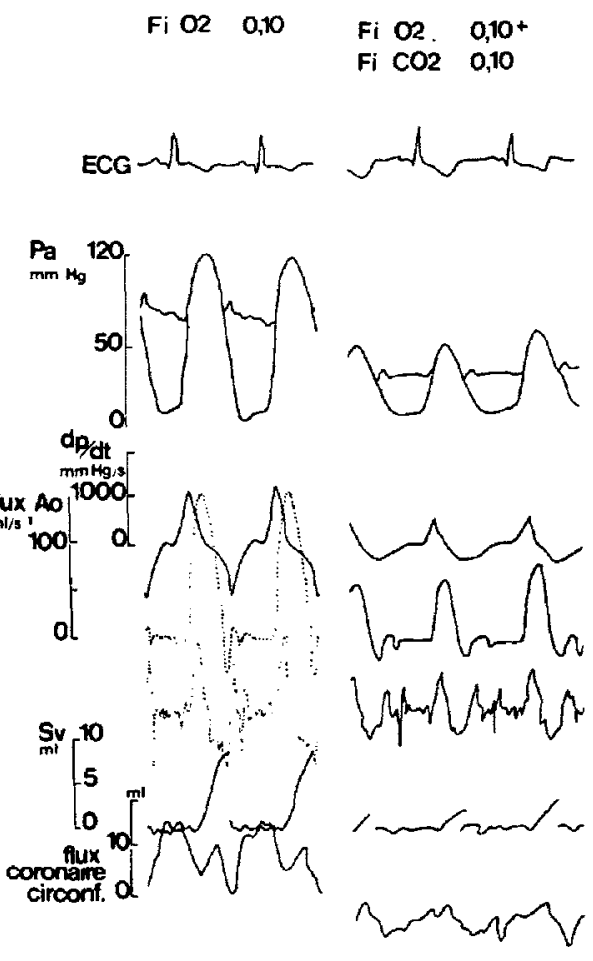

SCHEMA No5. Un chien subit sous Halothane à 0.8 pour cent une hypoxie, puis une hypercapnie. Tous ses paramètres circulatoires varient en même temps de façon péjorative. (Document obligeamment prêté par le Dr P. Foex.)

Sur un enregistrement réalisé par Pierre Foex (schéma $n^{0} 5$ ), cette pluralité d'action est évidente, au cours d'une épreuve d'asphyxie sous halothane à 0.8 pour cent, où toutes les constantes sont gravement altérées dans les mêmes délais: les ondes $\mathrm{T}$, les pressions, le $\mathrm{dP} / \mathrm{dt}$, le flux aortique, le volume d'éjection, le flux coronaire.

\section{LES INDICES HEMODYNAMIQUES VARIENT POUR DES CAUSES MULTIPLES ET SONT ASPECIFIQUES}

Ainsi, la tension artérielle est un produit qui varie comme les résistances systémiques et le débit. Elle peut rester stable quand ses composantes changent si les variations se compensent. On peut notamment constater l'immuabilité de la tension artérielle tandis que ses facteurs se modifient, par exemple, au cours de 24 heures d'anesthésie à l'alfatésine - fentanyl à débit constant chez huit chiens: les points de débit et de résistance systémique se déplacent, mais la ten- 


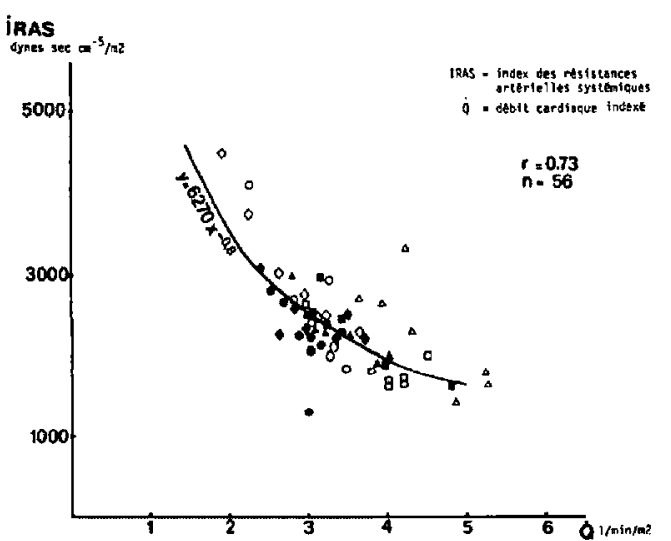

Schema N"6. Le nuage de points représente les variations de l'index cardiaque en fonction des IRAS, à TA quasi constante, chez huit chiens anesthésiés à débit régulier pendant 24 heures. Le nuage de points se place sur l'hyperbole de l'isobare TA.

$$
\left(y=6270 x^{-0.9}\right)
$$

Les symboles utilisés sont affectés à des animaux différents.

sion artérielle peut rester constante. Le nuage de points englobe l'isobare de tension artérielle qui effectivement a peu bougé, à la différence des valeurs inscrites sur le graphique (schéma $\pi^{\circ} 6$ ).

La pression veineuse centrale dépend simultanément du débit cardiaque et du remplissage vasculaire, comme l'a montré Guyton. ${ }^{7}$ Les courbes de fonction cardio-systémique l'objectivent: la PVC diminue quand augmente le débit. Mais pour un même débit, il y a deux PVC selon l'importance de la volémie (schéma $\mathrm{n}^{0} 7$ ).

Les courbes de fonction ventriculaire dépendent aussi de la post-charge, de la stimulation sympathique et de la fréquence cardiaque (schéma $n^{\circ} 8$ ). Filner ${ }^{5}$ rappelle qu'elles sont souvent plates sous anesthésie générale, du fait de l'effet inotrope négatif des substances anesthésiques, tandis que pour Sanghvi $i^{40}$ une augmentation volémique accroit la pré-charge mais diminue l'inotropie en stimulant les baro-récepteurs, donc en inhibant le tonus sympathique. D'où la difficulté d'interpréter les courbes de fonction ventriculaire dans les conditions opératoires où la volémie n'est pas constante.

La contractilité myocardique varie aussi selon les conditions circulatoires, puisque la vitesse de raccourcissement des fibres cardiaques augmente avec la précharge et diminue avec la postcharge. Le pic de $\mathrm{dP} / \mathrm{dt}$, lui, augmente avec la pré et la post-charge. Quelques indices dérivés y seraient moins sensibles (schèma $n^{\circ} 9$ ).
A
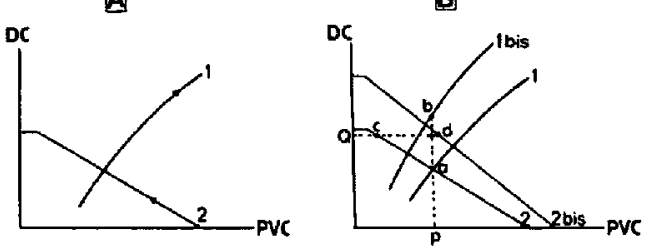

SCHEMA $N^{*} 7$. Sur ces graphiques empruntés à Guyton, on lit:

En $A:$ 1. que le débit cardiaque s'accroît quand la pression de remplissage (ici pression veineuse) augmente (lors d'une transfusion rapide par exemple) (courbe $n^{\circ} 1$ ). Cette relation illustre la loi de Starling et détermine la fonction ventriculaire. 2. que la PVC (courbe $n^{\circ}$ 2) diminue quand le débit augmente, par exemple lors de l'injection d'un cardiotonique.

$E n B:$ que l'hypervolémie remonte la courbe 2 en 2 bis et la stimulation sympathique remonte la courbe $I$ en I bis.

On dessine deux courbes extrêmes ( 1 et 1 bis) de fonction ventriculaire normale et deux courbes extrêmes ( 2 et 2 bis) de "fonction systémique" normale.

Ainsi, pour une mème PVC (p) on peut avoir deux débits cardiaques extrèmes (a et b) selon l'inotropie, et pour un même débit (Q), on peut avoir deux PVC différentes (c et d) selon la volémie.

$\mathrm{La} \mathrm{Vo}_{2}$ globale, mesurée ou calculée, ne rend compte des besoins réels tissulaires en oxygène que si la perfusion est suffisante et la teneur en hémoglobine aussi. Dans le cas contraire, elle ne reflète que ce qui peut être prélevé à l'extérieur et transporté jusqu'aux tissus. Les mesures faites tout au long des 24 heures d'anesthésie, déjà mentionnées, de huit chiens, en rendent compte: tandis que le débit reste constant pour une raison ignorée, la $\mathrm{VO}_{2}$ et l'extraction sanguine d'oxygène augmentent. Si la différence artério-

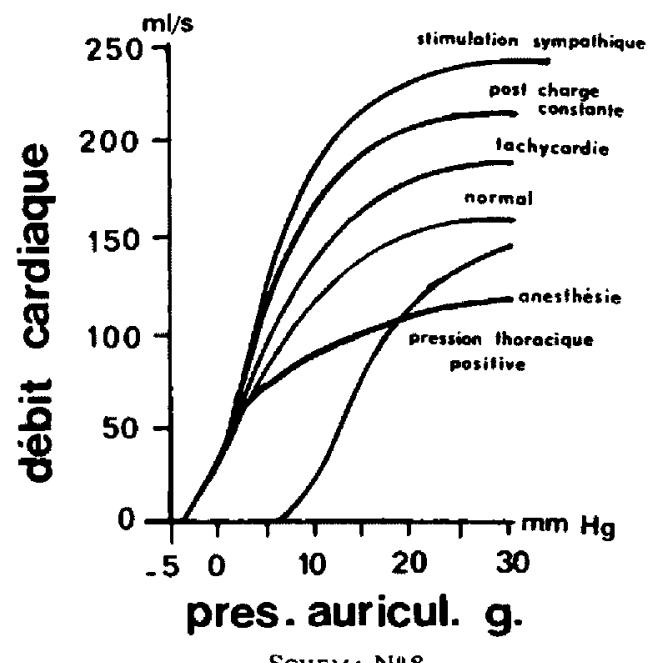

SCHEMA N' 8 

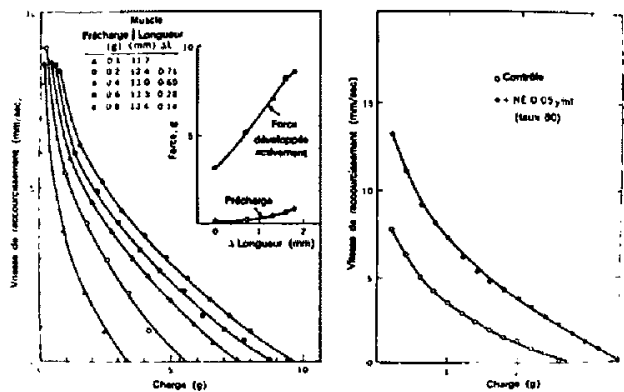

SCHEMa N*9. La vélocité de la contraction dépend de la pré et de la post-charge. $\mathrm{Vo}_{2}$ myocardique et vélocité ont ure relation positive. L'isoprénaline, les glucosides redressent les courbes de vélocité. Dans ce cas, la $\mathrm{VO}_{2}$ myocardique augmente.

veineuse augmentait encore, à débit constant, la $\mathrm{VO}_{2}$ calculée ou mesurée pourrait être inféricure aux besoins réels (schéma $\mathrm{n}^{\circ} 10$ ).

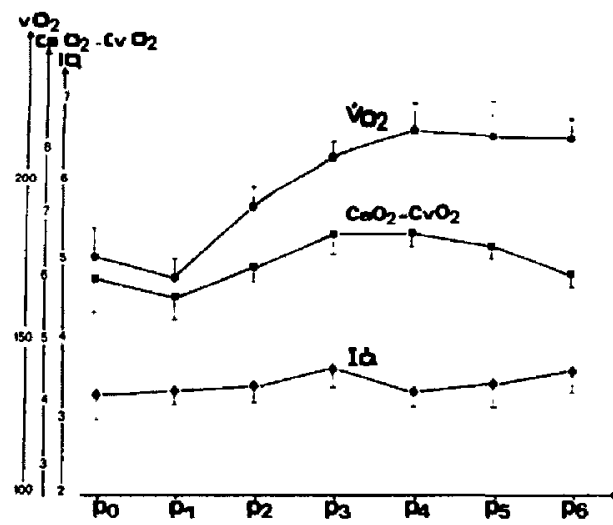

Schema No 10. Sur 24 heures, huit chiens anesthésiés à concentration régulière ont un débit cardiaque stable. Ici, l'élévation de la $\mathrm{VO}_{2}$ est compensée par une augmentation de l'extraction sanguine d'oxygène. Ainsi, les propriétés du sang lui-même permettent de tumponner les écarts entre débits et $\mathrm{Vo}_{2}$. (P. Girardet, et coll.).

Tous les paramètres hémodynamiques mesurables sont liés par une relation assez lâche, non quantifiée.

Cette formule (schéma $n^{0} \mathrm{~J} 1$ ) qui ne prétend pas à l'exactitude, a le mérite de montrer que l'on ne

\section{$\dot{V}_{2}$ myo $=f\left(W c, d P / d t, T, V_{\text {max }}\right)$ avec Wc $=$ SV $\times$ F $\times$ TA}

SChema Noll. Dans cette relation, les agents de la dynamique circulatoire sont mis en place.

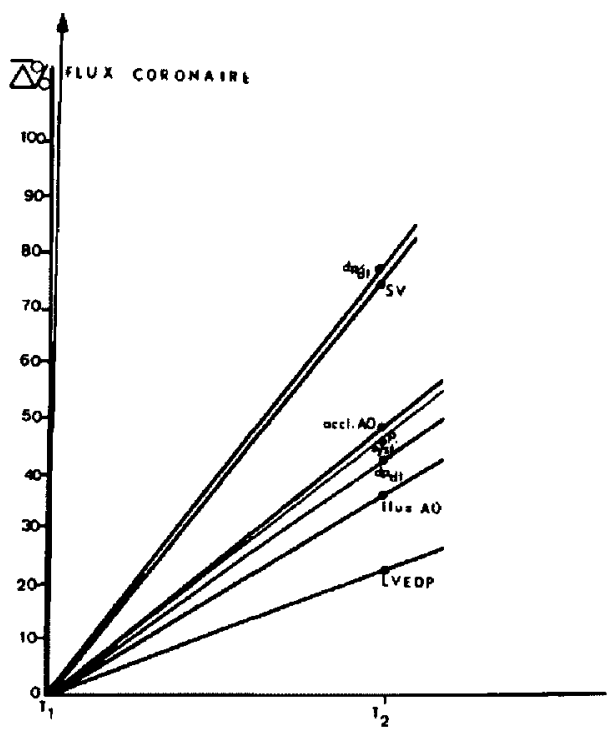

SCHEMA N* 12. Sur cette représentation des pour centages des variations provoquées par l'asphyxie du chien anesthésié dont on a vu les enregistrements hémodynamiques, on constate que la proportion de changement n'est pas la même. Tandis que le flux coronaire est devenu nul, la relaxation ventriculaire et le volume d'éjection sont plus altérés que les autres indices.

peut modifier totalement l'un des termes de l'équation sans en changer d'autres.

D'un autre côté, un seul des termes peut rester immuable quand d'autres changent, masquant les variations d'ensemble, comme l'arbre peut cacher la forêt.

Pour faire un diagnostic de situation hémody. namique, il est donc nécessaire:

(1) d'enregistrer au même moment plusieurs mesures simples ou composées et d'étudier le pourcentage de variations de chacune dentre elles (schéma $n^{\circ} 12$ ).

(2) d'observer leur évolution spontaneé dans le temps. ou sous épreuve de charge comme le fait Filner $^{5}$, et de dresser des diagrammes de tendance du type obtenu sur un écran cathodique au sortir d'un calculateur alimenté en données "élémentaires" (schéma ${ }^{\circ} 13$ ).

\section{Sur le plan pratique, il faut se DEMANDER QUELLE EST LA VALEUR PRONOSTIQUE DES RENSEIGNEMENTS} HEMODYNAMIQUES RECUEILLIS

Chez les patients atteints de cardiopathies, personne ne conteste l'aspect péjoratif d'une 


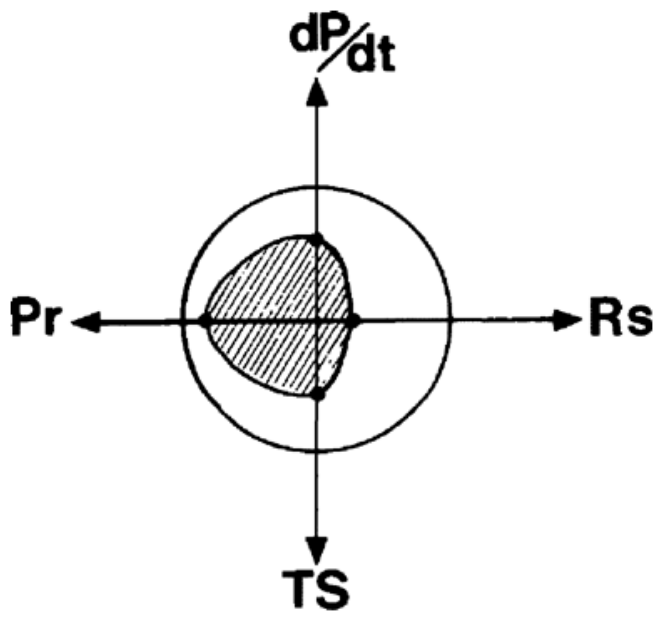

SChema No 13. Projection sur écran cathodique d'une surface circulaire déformée par les variations simultanées des résistances systémiques RS, d'une pression de remplissage $\mathrm{Pr}$, d'un indice de contractilité dP/dt, et du travail systolique TS. Le grand cercle représente la surface correspondant à des valeurs normales. La surface elliptoïde hachurée ce que l'on peut observer sous anesthésie halogénée stable. Ce genre de tracé peut être obtenu en continu par des appareils conmercialisés depuis plusieurs années.

augmentation de la pression capillaire bloquée par example, ou de l'augmentation de la période de pré-éjection; encore que, comme le rappelle Swan, ${ }^{9}$ le raisonnement ne soit pas toujours simple pour déterminer si le myocarde subit un préjudice au cours de ces altérations. Mais le sujet sain tolère curieusement la dépression circulatoire anesthésique dans la mesure où elle ne coincide pas avec une modification brutale de la pré ou de la post-charge.

La plupart des surdosages anesthésiques enrayés à temps n'entrainent pas de séquelles circulatoires.

Cela tient à trois raisons:

(1) il existe des réserves énergétiques de créatine phosphate dans le myocarde permettant 10 à 20 minutes d'hypoxie ischémique. Une partie de ces réserves est probablement utilisable quel que soit le blocage enzymatique possible;

(2) le surdosage déprime la contractilité, mais aussi le travail cardiaque, donc la $\mathrm{VO}_{2}$, épargnant en quelque sorte le myocarde, un peu comme le font les béta-bloquants. La demande baisse autant que l'offre.

Ceci explique qu'en cas de crise hémodynamique vers la baisse chez le sujet sain imprégné brusquement d'un dépresseur, l'indice EVR*

*'Endocardial viability rate". d'adaptation de perfusion coronaire à l'effort systolique et à la demande d'oxygène, ne soit pas effondré. Ceci est net sur le chien asphyxique montré précédemment (schéma n⿳05);

(3) le myocarde reste réactif aux stimulations sympathiques ou pharmacody namiques.

L'ultime moyen de connaitre la réelle "souffrance mycardique" au cours d'une dépression circulatoire serait-il l'observation biochimique du sang coronaire? La baisse continue de la $\mathrm{Po}_{2}$ et du $\mathrm{pH}$ du sinus traduisent la constitution d'une dette d'oxygène. Encore faut-il savoir à partir de quel moment elle est irréversible.

Dans cet esprit, Giuntinito met en place des sondes optiques pour apprécier en continu la $S v_{\mathrm{O}_{2}}$ myocardique chez des patients en menace $d$ 'infarctus. L'étude des iso-enzymes des CPK peut aussi donner quelques indications tardives, mais leur élévation traduit une altération définitive.

Au terme de cet exposé critique, trois idées pourraient ètre retenues:

(1) toute action pharmacodynamique entraine une réaction globale et modulée de la circulation;

(2) les indices hémodynamiques sont aspécifiques et d'interprétation délicate;

(3) une dépression circulatoire anesthésique de courte durée, aussi impressionnante soit-elle, est curieusement bien tolérée sur le plan myocardique.

\section{RÉSUMÉ}

Le praticien qui doit choisir des méthodes d'anesthésie et de surveillance hémodynamique adaptées à l'état de ses patients, cherche dans la littérature réponses à ses questions. Il n'y trouve pas toujours des notions utilisables dans son exercice courant.

En effet, la réaction circulatoire à un agent phairmaco-dynamique est diffuse et non spécifique, comme le rappelent divers exemples théoriques et pratiques. En particulier, il n'est pas sûr que diminuer la perfusion coronaire soit "mauvais" si la $\mathrm{VO}_{2}$ myocardique diminue en proportion.

D'autre part, les indices hémodynamiques (PA, PV, dP/dt) sont composés à partir de données élémentaires interdépendantes; il en résulte qu'ils sont eux aussi interdépendants.

Un dernier fait est troublant: les agents anesthésiques dépresseurs peuvent déclencher des crises hémodynamiques sévères mal supportées par les viscères en général mais, semble-t-il mieux par le myocarde qui ne souffre pas irrémédiablement si l'anoxie ischémique infligée ac- 
compagne une diminution brutale du travail cardique externe. Ceci explique la bonne tolérance cardiaque à un surdosage anesthésique passager.

Ii est difficile de déterminer quelles sont les premières modifications hémodynamiques duès à l'anesthésie que l'on doit traiter. La biochimie du sang veineux coronaire elle-même n'apporte pas aujourd'hui la solution.

\section{SUMMARY}

Physicians must choose the anaesthetics for their patients and select the methods to check their haemodynamic status. Experimental works do not always bring sufficient information to help them in their daily practice.

Circulatory reaction to a pharmacodynamic agent is diffuse and non-specific. Some examples drawn from theoretical considerations and practical situations support this view. The difficulty of assessing the actual damage caused by one apparent variation still remains. On the other hand, haemodynamic indices such as PA, CVP, dP/dt are composed of elementary data that are interdependent; so indices are interdependent, too.

A puzzling fact is that depressing anaesthetics can initiate severe haemodynamic crisis, generally badly tolerated by tissues, but better tolerated by myocardium which is not definitely altered if the anoxia inflicted is accompanied by a real decrease in cardiac work. This explains the often slight effect of short anesthetic overdosage.

Previous haemodynamic variations to be countered relative to anaesthesia are not really known. Biochemistry of the venous coronary blood does not yet bring striking features in this field.

\section{ABréviations}

$\mathrm{VO}_{2}=$ consommation d'oxygène $(\mathrm{ml} / \mathrm{min})$

$\mathrm{RS}=$ résistances systémiques (dynes $/ \mathrm{sec} / \mathrm{cm}^{-5}$ )

$\mathrm{dP} / \mathrm{dt}=$ indice de contractibilité

$\mathrm{PA}=$ pression artérielle $(\mathrm{mm} \mathrm{Hg})$

$\mathrm{IQ}=\mathrm{IC}=$ index cardiaque $(1 / \mathrm{min} / \mathrm{m} 2)$

$\mathrm{DC}=$ débit cardiaque $(\mathrm{I} / \mathrm{min})$

IRAS = index de résistances artérielles systémiques (dynes $/ \mathrm{sec} / \mathrm{cm}^{-5} / \mathrm{m} 2$ )

$\mathrm{PVC}=$ pression veineuse centrale $(\mathrm{mm} \mathrm{Hg})$

$\mathrm{SV}=$ volume systolique

$\mathrm{Pr}=$ pression de remplissage

$\mathrm{TS}=$ travail systolique

$\mathrm{EVR}=$ "endocardial viability rate"

$\mathrm{T}=$ tension pariétale du ventricule

$W=$ travail cardiaque
WIVG $=$ travail systolique ventriculaire gauche coron $=$ flux coronaire

$\mathrm{Fi}_{\mathrm{O}_{2}}=$ concentration d'oxygène inspiré

$\mathrm{Ca}_{\mathrm{O}_{2}}=$ contenu artériel en oxygène $(\mathrm{ml} / 100 \mathrm{ml}$ de sang)

$\mathrm{Cv}_{\mathrm{O}_{2}}=$ contenu veineux en oxygène $(\mathrm{ml} / 100 \mathrm{ml}$ de sang)

Accl $A O=$ accélération du flux aortique

\section{BIBLIOGRAPHIE}

1. Patschke, D., Bruckner, J.B., Eberlein, H.J., Hess, W., TARnow, J., \& Weymar, A. Effects of althesin, etomidate and fentanyl on haemodynamics and myocardial oxygen consumption in man. Can. Anaesth. Soc. J. 24: 57-69 (1976).

2. Merin, R.G., Kumazawa, T., \& Luka, N.L. Enflurane depresses myocardial function, perfusion and metabolism in the dog. Anesthesiology, 45: 501-507 (1976).

3. Dauchot, P.J., Rasmussen, J.P., Nicholson, D.H., Divers, R.T., Katona, P.G., Zollinger, R.M., KNOKE, J.D., KYo, E.W., \& GRAVENSTEIN, J.S. On-line systolic time intervals during anesthesia in patients with and without heart disease. Anesthe siology $44: 472-480$ (1976).

4. Byrick, R.J., Teasdale, S.J., \& Young, A. Systolic time interval changes after aorto-coronary bypass. Canad. Anaesth. Soc. J. 24: 175-185.

5. Fil.ner, B.E. \& Karliner, J.S. Alterations of normal left ventricular performance by general anesthesia. Anesthesiology, 45: 610-621 (1976).

6. Brutsaert, D.L. \& Sonnenblick, E.H. Cardiac muscle mechanics in the evaluation of myocardial contractility and pump function: problems, concepts and directions. Progress Cardiovasc. Dis. 16: 337-361 (1973).

7. Gurron, A.C., Jones, C.E., \& Coleman, T.G. Circulatory physiology: cardiac output and its regulation. W.B. Saunders Cy. Ed. Philadelphia, S56 pp. (1973).

8. Roberts, J.G., foex, P., Clakke, T.N.S., BenNETT, M.J., \& SANER, C.A. Haemodynamic interactions of high-dose propranolol pretreatment and anaesthesia in the dog. 3. The effects of haemorrhage during halothane and trichloroethylene anaesthesia. Brit. J. Anaesth. 48: 411418 (1976).

9. Swan, H.J.C., Forrester, J.S., Diamond, G.. Chatterjee, K., \& Parmley, W.W. Haemodynamic spectrum of myocardial infarction and cardiogenic shock. Circulation 45: 1097-1110 (1972).

10. Giuntin, C. Communication personnelle.

11. Braunwald, E. On the difference between the heart's output and its contractile state. Circulation 43: 171-174 (1971).

12. Braunwald, E. Control of myocardial oxygen consumption. Physiologic and clinical considerations. Amer. J. Cardiol., 27: 416-432 (1971).

13. Braunwald, E. Current concepts in cardiology. Determinants and assessment of cardiac function. New Engl. J. Med. 296: 86-89 (1977).

14. Cross, C.E., Rieben, P.A., Barron, C.I., \& SALISBURY, P.F. Effects of arterial hypoxia on the 
heart and circulation: an integrative study. Amer. J. Physiol., 205: 963-970 (1963).

15. Diaz, F.A., Bianco, J.A., Bello, A., Beer, N. Velarde, H., Izquierdo, J.P., \& Jaen, R. Effects of ketamine on canine cardiovascular function. Brit. J. Anaesth, 48: 941-945 (1976).

16. Foex, P. \& Prys-Roberts, C. Interactions of beta-receptor blockage and $\mathrm{PCO}_{2}$ levels in the anaesthetized dog. Brit. J. Anaesth., 46: 397-405 (1974).

17. Gersh, B.J., Prys-Roberts, C., Reuben, S.R., \& SCHulTz, D.L. The effects of halothane on the interactions between myocardial contractility, aortic impedance and left ventricular performance. 2. Aortic imput impedance and the distribution of energy during ventricular ejection. Brit. J. Anaesth., 44: 767-774 (1972).

18. Givdicelli, J.F. Pharmacologie cardiaque. Asclepios Ed., Paris, 279 pp. (1976).

19. GorLIn, R. Current concepts in cardiology. Practical cardiac hemodynamics. New Engl. J. Med. 296: 203-205 (1977).

20. Graham, T.P., Covell, J.W., Sonnenblick, E.H., Ross, J., \& Braunwald, E. Control of myocardial oxygen consumption: relative influence of contractile state and tension development. J. Clin. Invest. 47: 375-385 (1968).

21. Guyton, A.C. Regulation of cardiac output. New Engl. J. Med. 277: 805-812 (1967)

22. Harlan, W.R., Oberman, A., Grimm, R., \& RosATI, R.A. Chronic congestive heart failure in coronary artery disease: clinical criteria. Ann. Internal. Med. 86: 133-138 (1977).

23. HJLL, D.W. \& MERrifield, A.J. Left ventricular ejection and the Heather index measured by noninvasive methods during postural changes in man. Acta Anaesth. Scand. 20:313-320 (1976).

24. Kanter, S.F. \& Samuels, S.I. Anesthesia for major operations on patients who have transplanted hearts. A review of 29 cases. Anesthesiology 46: 65-68 (1977).

25. Lappas, D.G., Buckley, M.J., Laver, M.B. DaggetT, W.M., \& Lowenstein, E. Left ventricular performance and pulmonary circulation following addition of nitrous oxide to morphine during coronary artery surgery. Anesthesiology, $43: 61-69(1975)$.

26. LEVY, M.N. The cardiovascular physiology of the criticaliy ill patient. Surg. Clin. North Amer., 55: 483-499 (1975).

27. Marino, R.J., Romagnoli, A., \& Keats, A.S. Selective venoconstriction by Dopamine in comparison with Isoproterenol and Phenylephrine. Anesthesiology, 43: 570-572 (1975).

28. Marshall, M. Williams, W.G., Creighton, R.E., Vol,gyesı, G.A., \& STEWARd, D.J. A technique for measuring regional myocardial blood flow and its application in determining the effects of hyperventilation and halothane. Canad. Anaesth. Soc. J. 23: 244-251 (1976).

29. Mason, D.T., Spann, J.F., \& Zelis, R. Quantification of the contractile state of the intact human heart. Amer. J. Cardiol. 26: 248-257 (1970).

30. Michalot, G. Oxygénation extra-corporelle d'as- sistance dans l'hypoxémie réfractaire. Etude expérimentale de la voie de réinjection aortique rétrograde. Données gazométriques et hémodynamiques. Thèse de Medecine, Grenoble (1977).

31. Nandan, R.M. Action du Nitroprussiate en neurochirurgie. Thèse de Medecine, Grenoble. Sous presse

32. Parisi, A.F., Tow, D.E., Felix, W.R., \& SasaHARA, A.A. Noninvasive cardiac diagnosis. New Engl. J. Med. 296: 427-432 (1977).

33. Payne, R.M., HoRwitz, L.D., \& Mullins, C.B. Comparison of isometric exercise and angiotensin infusion as stress test for evaluation of left ventricular function. Amer. J. Cardiol. 3l: 428-433 (1973),

34. Philips, P.A., Marty, A.T., Miyamoto, A.M. A clinical method for detecting subendocardial ischemia after cardio-pulmonary bypass. J. Thor. Cardiovasc. Surg. 69: 30-39 (1975).

35. Prys-Roberts, C., Greene, L.T., Meloche, R., \& FOEX, P. Studies of anaesthesia in relation to hypertension. Hemodynamic consequences of induction and endotracheal intubation. Brit. J. Anaesth. 43: 531-547(1971)

36. Roberts, J.G., Foex, P., Clarke, T.N.S., \& BENNETT, M.J. Haemodynamic interactions of high-dose propranolol pretreatment and anaesthesia in the dog. 1. Halothane dose-response studies. Brit. J. Anaesth. 48: 315-324 (1976).

37. Roberts, J.G., Foex, P., Clarke, T.S.N., Prys-Roberts, C., \& BennetT, M.J. Haemodynamic interactions of high-dose propranol pretreatment and anaesthesia in the dog. 2. The effects of acute arterial hypoxae mia at increasing depths of halothane anaesthesia. Brit. J. Anaesth. 48: 403-409 (1976).

38. Rolzen, M.F., Moss, J., Henry, D.P., \& Kopin, I.J. Effects of halothane on plasma catecholamines. Anesthesiology 41: 432-439 (1974),

39. Sarnoff, S.J., Baunwald, E., Welch, G.H., Case, R.B., Stainsby, W.H., \& Macrez, R. Hemodynamic determinants of oxygen consumption of the heart with special reference to tension time index. Amer. J. Physiol., 192: 148-155(1958).

40. Sanghvi, V.R., Khaja, F., Mark, A.L., Parker, J.P. Effects of blood volume expansion on left ventricular haemodynamics in man. Circulation 46: 708-787 (1972).

41. Smith, G. The coronary circulation and anaesthesia. Brit. J. Anaesth. 48: 933-934 (1976).

42. Starling, E.H. The linacre lecture on "the Law of the heart". London and New York: Longman, Green and Co. (1918).

43. SWAN, H.J.C. \& GANZ, W. Use of balloon flotation catheters in criticially ill patients. Surg. Clinics North Amer. 55: 501-520 (1975).

44. Tomlin, P.J. \& Duck, F.A. Transoesophageal aortic velography in man. Canad. Anaesth. Soc.J. 22: 561-571 (1975).

45. WeXler, L.F. \& Pohost, G.M. Hemodynamic monitoring: noninvasive techniques. Anesthesiology 45: 156-183 (1976).

46. YANG, S.S. From cardiac catheterisation data to hemodynamics parameters. Davis Ed., Philadelphia, 307 pp. (1972). 